\title{
Joint Resource Allocation and Route Selection for Multi-hop Cognitive Relay Networks
}

\author{
Yuanpeng Gao; Ling Wang; Rong Chai \\ Key Lab of Mobile Communication Technologies \\ Chongqing University of Posts and Telecommunications \\ China
}

\begin{abstract}
In this paper, a multi-hop cognitive relay network consisting of multiple PU transmission pairs, SU transmission pairs and relay SUs is considered. To achieve the performance enhancement of multi-hop transmission links between SU pairs, an energy efficient constrained shortest path first (CSPF)based joint resource allocation and route selection algorithm is proposed, which consists of two sub-algorithms, i.e., CSPFbased route selection sub-algorithm and energy efficiencybased resource allocation sub-algorithm. More specifically, we first apply CSPF-based route selection sub-algorithm to obtain the shortest transmission route under the transmission constraints. Then, an energy efficiency-based resource allocation problem of the shortest routes is formulated and solved by applying iterative algorithm and Lagrange dual method. Finally, the energy efficiency of the shortest transmission routes is examined and the globally optimal route selection and resource allocation strategy is obtained which offers the maximal energy efficiency of the transmission route. Simulation results demonstrate the effectiveness of the proposed algorithm.
\end{abstract}

\section{KEYWORDS}

Cognitive network, multi-hop relay, resource allocation, route selection, energy efficiency

\section{ACM Reference format:}

Yuanpeng Gao; Ling Wang; Rong Chai. . Joint Resource Allocation and Route Selection for Multi-hop Cognitive Relay Networks. In Proceedings of MobiMedia 2017, Chongqing, China, July 2017, 8 pages.

DOI:

\section{INTRODUCTION}

The rapid development of mobile broadband services with continuously increasing traffic volumes has posed requirements on available frequency spectrum. However, traditional static spectrum allocation policy has resulted in the exhaustion of licensed spectrum while on the other hand a lot of allocated licensed spectrum is extremely under utilized.

Permission to make digital or hard copies of all or part of this work for personal or classroom use is granted without fee provided that copies are not made or distributed for profit or commercial advantage and that copies bear this notice and the full citation on the first page. To copy for personal or classroom use is granted without fee provided that otherwise, to republish, to post on servers or to redistribute to lists, requires prior specific permission and/or a fee.

MOBIMEDIA 2017, July 13-14, Chongqing, People's Republic of China

Copyright () 2017 EAI 978-1-63190-156-0 stress this problem, cognitive radio networks (CRNs) have been proposed which allow unlicensed users referred to as secondary users (SUs) to opportunistically utilize the licensed bands of licensed users, i.e., primary users (PUs) without affecting the normal communications of the PUs [1]. In CRNs, source and destination SU pairs may conduct information interaction with each other. In the case that the direct transmission link between one source SU and its destination SU is unavailable, relay technology can be applied through which one or multiple relay SUs are selected to help forwarding data packets for the SU pair. To achieve the transmission performance enhancement of multi-hop SU pairs, the route selection and resource allocation schemes have to be considered.

In recent years, there are some research works focusing on resource allocation problems in CRNs. In [2], the uplink transmission in a CRN consisting of a single PU and multiple SUs is considered. The authors propose a dynamic scheduling and power-allocation policy that provides the required average delay guarantees to all SUs and protects the PU from harmful interference. In [3], the power allocation problem is investigated in CRNs which employing non-orthogonal multiple access (NOMA) technique. The authors propose a power allocation algorithm, which fully exploits the characteristics of NOMA-based system and aims to maximize the number of admitted SUs under the maximum power and interference constraints. In [4], the authors address the channel allocation problem for multi-channel cognitive vehicular networks with the objective of maximizing system-wide throughput. Since the formulated problem is an NP-hard non-linear integer programming problem, the authors propose a probabilistic polynomial-time-approximation algorithm based on linear programming and then develop a deterministic constant factor approximation algorithm with a more favorable time complexity. In [5], the authors consider cluster-based underlay multi-hop CRNs and propose an ad-hoc routing protocol, namely, the highest transmit power relay selection (HTPRS) protocol and its improved version. The exact endto-end outage probability of the networks when employing the two routing protocols is derived by taking into account both peak-power and peak-interference constraints.

In [6], the authors present a framework for distributively optimizing the transmission strategies of SUs in an ad hoc CRN and design an optimal transmit power and channel access probability strategy for the SUs which maximizes the number of SU transmissions per unit area. In [7], the authors investigate dynamic channel and rate selection in 
CRNs where the SU transmitters are allowed to change the selected channel and rate pair in order to opportunistically track the pair offering the highest throughput. The authors formulate the problem of sequential channel and rate selection as an online optimization problem and derive fundamental performance limits and propose algorithms that achieve these limits.

Some research works jointly consider route selection and resource allocation problems of CRNs. In [8], the authors study the robust relay selection and power allocation problem for OFDM-based cooperative CRNs with channel uncertainties. By characterizing the channel uncertainties as ellipsoid set and interval set, a semi-infinite programming problem is formulated to maximize the capacity of the network and the optimal joint relay selection and power allocation strategy can be obtained by solving the optimization problem. In [9], the authors investigate the joint channel allocation and routing problem in cognitive wireless mesh networks and propose an economic framework for adaptation and control of the network resources with the goal of network profit maximization. The authors in [10] study the joint routing and time-frequency resource allocation problem in cognitive radio based wireless mesh networks and propose a joint optimization algorithm which minimizes the aggregate end-toend delay of all the network flows.

Previous research works on route selection and resource allocation in multi-hop CRNs mainly focus on joint relay selection and time/frequency resource allocation, few works consider the optimization of the end-to-end transmission performance of the entire routes, which may result in the suboptimal transmission performance due to the lack of global routing and resource information. Furthermore, while the performance optimization of the data rate and the propagation delay of the transmission links is taken into account in previous works, few of them jointly considers the data rate and power consumption of the transmission nodes, which is highly undesired especially for battery sensitive user devices.

In this paper, an energy efficient constrained shortest path first (CSPF)-based joint resource allocation and route selection algorithm is proposed which consists of two sub-algorithms, i.e., CSPF-based route selection sub-algorithm and energy efficiency-based resource allocation sub-algorithm.

The rest of this paper is organized as follows. The system model is discussed in Section 2. In Section 3, the proposed CSPF-based route selection algorithm is presented. Energy efficiency optimization problem formulation is described in Section 4. Solution to the optimization problem is discussed in Section 5 and simulation results are given in Section 6 . Finally, we conclude this paper in Section 7.

\section{SYSTEM MODEL}

In this paper, we consider a CRN in which PUs transmit their information to primary base station (PBS) on licensed spectrum, and a number of SU transmission pairs are allowed to transmit data packets in an ad-hoc mode. Assume that

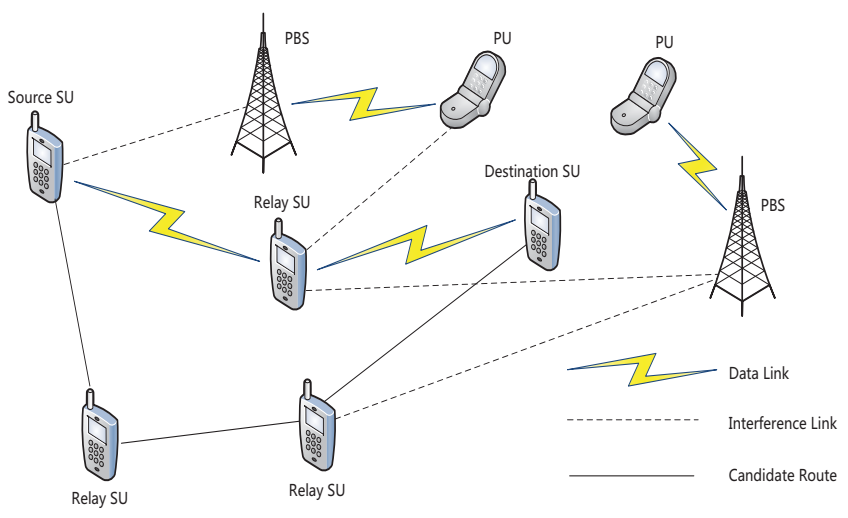

Figure 1: System model

each PU is allocated one licensed channel for data transmission and each licensed channel is only assigned to one $\mathrm{PU}$, hence, no spectrum competition and transmission interference exist between PUs. For convenience, we assume the number of the PUs is same as the number of the channels and the $k$ th $\mathrm{PU}$ is allocated the $k$ th channel for data transmission, $1 \leq k \leq K$, where $K$ denotes the number of the PUs and that of the channels.

To improve spectrum efficiency, SU pairs are allowed to share the spectrum resources with the PUs. More specifically, we assume that underlay spectrum sharing mode is applied between PUs and SUs, i.e., PUs and SUs may transmit simultaneously on the same licensed channel provided that the transmission performance of both the PUs and the SUs can be guaranteed. In the case that the destination $\mathrm{SU}$ is out of the transmission range of the source $\mathrm{SU}$, one or multiple relay $\mathrm{SU}(\mathrm{s})$ can be applied to forward the data packets for the SU pair. In this paper, it is assumed that decode-and-forward (DF) mode is employed in the relay SUs. Fig. 1 shows the system model considered in this paper.

For a SU pair, in the case that multiple candidate routes are available, optimal route selection has to be conducted. In addition, for both the source SU and relay SUs, channel and transmit power allocation strategy should be designed in an optimal manner so that the transmission performance of the SU pair can be enhanced. In this paper, we study the route selection and resource allocation problem of one SU pair and design an optimal joint route selection, and channel and transmit power allocation strategy for the source SU and the relay SUs.

\section{CSPF-BASED ROUTE SELECTION ALGORITHM}

For a given SU pair, a large number of transmission hops may result in long transmission delay and large resource overhead, which are highly undesired. Thus, the transmission hop of the candidate routes should be considered in designing optimal route selection algorithm. Furthermore, as each SU pair may have certain QoS requirements, the selected routes may have to meet some transmission constraints. In this section, 
we jointly consider the number of hops and the constraints of the transmission routes, and propose a CSPF-based route selection sub-algorithm that selects the candidate shortest routes which meet the route selection constraints.

\subsection{Constraints on the Transmission Routes}

For a given source and destination SU pair, we apply routing discovery algorithm and select all possible routes between the SU pair. Let $\Psi_{0}$ denote the set containing all the possible routes between the SU pair and $\psi_{0}^{(i)}$ denote the $i$ th route between the SU pair, $1 \leq i \leq N^{\text {tot }}$, where $N^{\text {tot }}$ denotes the total number of the possible routes. We further denote $R^{(i)}$ as the data rate of the $i$ th possible connecting route, (for simplicity, also referred to as the $i$ th route hereafter), , and denote the data rate of the $l$ th hop of the $i$ th route as $R_{l}^{(i)}$, $1 \leq l \leq L_{0}^{(i)}$, where $L_{0}^{(i)}$ denotes the number of hops of the $i$ th route, we obtain

$$
R^{(i)}=\min _{1 \leq l \leq L_{0}^{(i)}, \psi_{0}^{(i)} \in \Psi_{0}}\left\{R_{l}^{(i)}\right\} .
$$

In this paper, user QoS requirement on transmission rate is stressed and the data rate of the transmission route is considered as the QoS metric of the SUs, i.e., each SU pair is assumed to have a minimum data rate requirement. Denoting $R^{(\mathrm{s}, \mathrm{min})}$ as the minimum data rate requirement of the SU pair, the $i$ th route has to meet data rate constraint, i.e., $R^{(i)} \geq R^{(\mathrm{s}, \mathrm{min})}$, which is equivalent to the following condition:

$$
R_{l}^{(i)} \geq R^{(\mathrm{s}, \mathrm{min})}, 1 \leq l \leq L_{0}^{(i)} .
$$

Let $\alpha_{l, k}^{(i)}$ denote the route selection and channel allocation variable of the $l$ th hop of the $i$ th route, i.e., $\alpha_{l, k}^{(i)}=1$ represents the $l$ th hop of the $i$ th route chooses the $k$ th channel for data transmission, otherwise, $\alpha_{l, k}^{(i)}=0$. Denoting $R_{l, k}^{(i)}$ as the corresponding data rate, $R_{l}^{(i)}$ can be expressed as

$$
R_{l}^{(i)}=\sum_{k=1}^{K} \alpha_{l, k}^{(i)} R_{l, k}^{(i)}
$$

Jointly considering (2) and (3), we can obtain that following constraint:

$$
R_{l, k}^{(i)} \geq R^{(\mathrm{s}, \min )} .
$$

According to Shannon's formula, $R_{l, k}^{(i)}$ can be calculated as:

$$
R_{l, k}^{(i)}=W \log \left(1+\frac{P_{l, k}^{(i)} h_{l, k}^{(i)}}{P_{k}^{(\mathrm{p})} g_{l, k}^{(\mathrm{p}, i)}+\sigma^{2}}\right)
$$

where $W$ denotes the bandwidth of the $k$ th channel, which is assumed to be identical for all the channels, $h_{l, k}^{(i)}$ and $P_{l, k}^{(i)}$ denote the channel gain and the transmit power of the $l$ th hop transmit SU of the $i$ th path on the $k$ th channel, $P_{k}^{(\mathrm{p})}$ denotes the transmit power of the $k$ th PU, $g_{l, k}^{(\mathrm{p}, i)}$ denotes the link gain from the $k$ th $\mathrm{PU}$ to the $l$ th hop receiving node of the $i$ th route on the $k$ th channel and $\sigma^{2}$ denotes the power of the channel noise.
Jointly considering (4) and (5), we can transform the data rate constraint in (4) equivalently into following transmit power constraint, i.e.,

$$
P_{l, k}^{(i)} \geq\left(2^{R^{(\mathrm{s}, \mathrm{min})} / W}-1\right) \frac{P_{k}^{(\mathrm{p})} g_{l, k}^{(\mathrm{p}, i)}+\sigma^{2}}{h_{l, k}^{(i)}} .
$$

As the parameters contained in the right side of (6) are assumed to be constants in this paper, (6) gives a fixed lower bound of $P_{l, k}^{(i)}$. On the other hand, for practical applications, user devices may have different maximum permissible transmit power. Denoting $P_{l}^{(i, \max )}$ as the maximum transmit power of the $l$ th hop transmit SU of the $i$ th route, the maximum power constraint can be expressed as

$$
P_{l, k}^{(i)} \leq P_{l}^{(i, \max )}, 1 \leq l \leq L_{0}^{(i)}, 1 \leq k \leq K .
$$

Jointly considering (6) and (7), we can obtain

$$
\left(2^{R^{(\mathrm{s}, \mathrm{min})} / W}-1\right) \frac{\sigma^{2}+P_{k}^{(\mathrm{p})} g_{l, k}^{(\mathrm{p}, i)}}{h_{l, k}^{(i)}} \leq P_{l}^{(i, \max )} .
$$

The above condition indicates that the $l$ th link of the $i$ th route can be selected as the candidate route of the SU pair on the $k$ th channel if only (8) holds, hence, we can obtain the candidate route set of the SU pair, denoted as $\Psi_{1}$, which can be expressed as

$\Psi_{1}=\left\{\psi_{0}^{(i)} \mid \psi_{0}^{(i)} \in \Psi_{0}, \forall 1 \leq l \leq L_{0}^{(i)}, \exists 1 \leq k \leq K,(8)\right.$ holds $\}$.

\subsection{Selecting the Shortest Candidate Routes}

For all the candidate routes collected in $\Psi_{1}$, the number of hops is examined and the candidate routes with the minimum number of hops are selected. Denote $N_{1}$ as the size of $\Psi_{1}$, i.e., the total number of the candidate routes, and denote $\psi_{1}^{(i)}$ as the $i$ th candidate route in $\Psi_{1}, L^{(i)}$ as the number of hops of the $i$ th candidate route, the minimum hop of all the candidate routes can be calculated as:

$$
L_{\min }=\min _{1 \leq i \leq N_{1}, \psi_{1}^{(i)} \in \Psi_{1}}\left\{L^{(i)}\right\}
$$

Collecting the candidate routes with the number of hops being $L_{\min }$ into a set $\Psi$, we obtain,

$$
\Psi=\left\{\psi_{1}^{(i)} \mid L^{(i)}=L_{\min }, \psi_{1}^{(i)} \in \Psi_{1}, 1 \leq i \leq N\right\} .
$$

We denote $\psi^{(i)}$ as the $i$ th shortest candidate route (SCR) in $\Psi$.

\subsection{Energy Efficiency Optimization-based Route Selection}

In this paper, to stress the importance of both the transmission rate and power consumption of the SCRs and to achieve the tradeoff of the two parameters, the energy efficiency of the SCRs collected in $\Psi$ is examined and optimized in terms of the transmit power and channel allocation strategy of the source and relay SUs, the detail optimization procedure will be discussed in following two subsections. 
Denote $\eta^{(i)}$ and $\eta^{(i, *)}$ as the energy efficiency of the $i$ th SCR and the corresponding optimal value, denote $\alpha_{l, k}^{(i, *)}$ and $P_{l, k}^{(i, *)}$ as the optimal channel allocation variable and power allocation variable of the $l$ th link of the $i$ th SCR when the $k$ th channel is allocated, we obtain

$$
\eta^{(i, *)}\left(\alpha_{l, k}^{(i, *)}, P_{l, k}^{(i, *)}\right)=\max _{\alpha_{l, k}^{(i)}, P_{l, k}^{(i)}}\left(\eta^{(i)}\right), 1 \leq i \leq N
$$

where $N$ denotes the number of the SCRs. Examining $\eta^{(i, *)}$ for all the $\psi^{(i)}$ in $\Psi$, the route $\psi^{(i, *)}$ which achieves the maximum energy efficiency can then be selected as the optimal route, i.e.,

$$
\psi^{(i, *)}=\underset{\psi^{(i)} \in \Psi}{\operatorname{argmax}}\left(\eta^{(i, *)}\right), 1 \leq i \leq N .
$$

The optimal power and channel allocation strategy of $\psi^{(i, *)}$ can also be obtained through the energy efficiency optimization. For a given $\operatorname{SCR} \psi^{(i)}, 1 \leq i \leq N$, the detail energy efficiency optimization procedure will be given in following section.

\section{ENERGY EFFICIENCY OPTIMIZATION PROBLEM FORMULATION}

In this section, we formulate the optimal power and channel allocation problem of the $i$ th SCR as an energy efficiency optimization problem.

\subsection{Energy Efficiency Modeling}

The energy efficiency of the $i$ th SCR can be defined as

$$
\eta^{(i)}=\min _{1 \leq l \leq L_{\min }}\left\{\eta_{l}^{(i)}\right\}
$$

where $\eta_{l}^{(i)}$ denotes the energy efficiency of the $l$ th link of the $i$ th $\mathrm{SCR}, 1 \leq l \leq L_{\mathrm{min}}$, and can be calculated as

$$
\eta_{l}^{(i)}=\sum_{k=1}^{K} \alpha_{l, k}^{(i)} \eta_{l, k}^{(i)}
$$

where $\eta_{l, k}^{(i)}$ denotes the energy efficiency of the $l$ th link of the $i$ th SCR when transmitting on the $k$ th channel, and can be expressed as

$$
\eta_{l, k}^{(i)}=\frac{R_{l, k}^{(i)}}{P_{l, k}^{(i)}+P_{\text {cir }}}
$$

where $P_{\text {cir }}$ denotes the circuit power consumption of the $l$ th hop transmitter of the $i$ th SCR when transmitting on the $k$ th channel. Without loss of generality, $P_{\text {cir }}$ is assumed to be a constant for all the transmitters in this paper.

\subsection{Optimization Constraints}

To achieve the maximum energy efficiency of the $i$ th SCR, $1 \leq i \leq N$, the following optimization constraints have to be considered.

C1: The maximum transmit power constraint
Due to the hardware limitation, the source and relay SUs of the $l$ th hop of the $i$ th SCR should meet the maximum transmit power constraint, $1 \leq l \leq L_{\mathrm{min}}$, i.e.,

$$
P_{l, k}^{(i)} \leq P_{l}^{(i, \max )}, 1 \leq k \leq K .
$$

C2: The minimum data rate constraint of the PUs

In CRN, due to the high priority of the PUs, the SUs can only access the licensed spectrum provided that the transmission requirements of the PUs can be guaranteed. Assuming each PU should meet a minimum data rate constraint, i.e.,

$$
R_{k}^{(p)} \geq R_{k}^{(\mathrm{p}, \min )}
$$

where $R_{k}^{(\mathrm{p})}$ denotes the data rate of the $k$ th PU and $R_{k}^{(\mathrm{p}, \mathrm{min})}$ denotes the minimum data rate requirement of the $k$ th $\mathrm{PU}$, $1 \leq k \leq K$. In the case that the source or relay SUs on the $l$ th hop of the $i$ th SCR share the channel with the $k$ th PU, $R_{k}^{(\mathrm{p})}$ can be expressed as

$$
R_{k}^{(p)}=W \log \left(1+\frac{P_{k}^{(\mathrm{p})} h_{k}^{(\mathrm{p})}}{\sigma^{2}+P_{l, k}^{(i)} g_{l, k}^{(i, \mathrm{p})}}\right)
$$

where $h_{k}^{(\mathrm{p})}$ denotes the channel gain of the link from the $k$ th $\mathrm{PU}$ to the PBS, $g_{l, k}^{(i)}$ denotes the channel gain of the link from the transmitter of the $l$ th hop of the $i$ th SCR to the PBS, $1 \leq l \leq L_{\min }$. Combining (18) and (19), we can obtain the transmit power constraint of the SUs, i.e.,

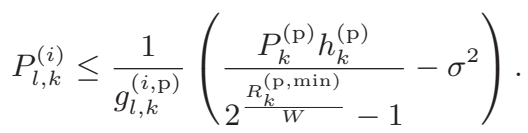

C3: The minimum data rate constraint of the SU pair

As the $i$ th SCR should meet the minimum data rate constraint, i.e., $R^{(i)} \geq R^{(s, \min )}$, all the links of the route should also meet the data rate constraint, i.e.,:

$$
\sum_{k=1}^{K} \alpha_{l, k}^{(i)} R_{l, k}^{(i)} \geq R^{(\mathrm{s}, \mathrm{min})}, 1 \leq l \leq L_{\mathrm{min}} .
$$

C4: Binary constraint on channel allocation variables

In this paper, the channel allocation identifiers are defined as binary variables, i.e.,

$$
\alpha_{l, k}^{(i)}=\{0,1\}, 1 \leq l \leq L_{\min }, 1 \leq k \leq K .
$$

\section{C5: Channel allocation constraint}

Due to hardware constraints, we assume that at most one channel can be allocated to the transmitter of the $l$ th hop of the $i$ th SCR, hence we obtain

$$
\sum_{k=1}^{K} \alpha_{l, k}^{(i)} \leq 1,1 \leq l \leq L_{\min }
$$




\subsection{Optimization Problem Formulation}

The energy efficiency of the $i$ th SCR can be optimized based on the given constraints, $1 \leq i \leq N$, and the optimization problem can be modeled as follows

$$
\begin{aligned}
& \max _{\alpha_{l, k}^{(i)}, P_{l, k}^{(i)}} \eta^{(i)} \\
& \text { s. t. C1 : } P_{l, k}^{(i)} \leq P_{l}^{(i, \max )}, \\
& \mathrm{C} 2: P_{l, k}^{(i)} \leq \frac{1}{g_{l, k}^{(i, \mathrm{p})}}\left(\frac{P_{k}^{(\mathrm{p})} h_{k}^{(\mathrm{p})}}{2^{\frac{R_{k}^{(\mathrm{p}, \mathrm{min})}}{W}}-1}-\sigma^{2}\right) \text {, } \\
& \text { C3 : } \sum_{k=1}^{K} \alpha_{l, k}^{(i)} R_{l, k}^{(i)} \geq R^{(\mathrm{s}, \min )}, \\
& \text { C4 : } \alpha_{l, k}^{(i)}=\{0,1\}, 1 \leq l \leq L_{\min } \text {, } \\
& \text { C5 : } \sum_{k=1}^{K} \alpha_{l, k}^{(i)} \leq 1 \text {. }
\end{aligned}
$$

It is apparent that to maximize $\eta^{(i)}$ in (39) is equivalent to maximizing the energy efficiency among all the links of the $i$ th SCR. Furthermore, as the data transmission of various links along the $i$ th SCR occupies different time durations, no specific constraints need to be considered for channel allocation among various links. Therefore, the resource allocation of each link can be conducted independently, the optimal energy efficiency of the $i$ th SCR can then be obtained based on the optimal solution of each link.

For the $l$ th link of the $i$ th $\mathrm{SCR}, 1 \leq l \leq L_{\text {min }}, 1 \leq i \leq N$, the optimization problem can be formulated as

$$
\begin{aligned}
& \max _{\alpha_{l, k}^{(i)}, P_{l, k}^{(i)}} \eta_{l}^{(i)} \\
& \text { s.t. } \mathrm{C} 1 \sim \mathrm{C} 5 \text { in }(39) .
\end{aligned}
$$

\section{SOLUTION TO THE OPTIMIZATION PROBLEM}

In this section, the optimization problem formulated in (25) is solved to obtain the optimal power and channel allocation strategy for the $l$ th link of the $i$ th SCR, $1 \leq l \leq L_{\min }$. As the optimization problem formulated in (25) involves the coupling of binary optimization and nonlinear fractional optimization, which cannot be solved conveniently using traditional optimization tools. Indeed, it can be shown that the power allocation for any given channel allocation strategy can be conducted independently, hence, the joint optimization problem can be equivalently transformed into power allocation subproblem and channel selection subproblem. More specifically, by formulating and solving the power allocation subproblem, the optimal transmit power can be designed and the corresponding energy efficiency can be obtained for each channel allocation strategy, then the optimal channel which corresponds to the maximum energy efficiency can be selected through solving the channel selection subproblem.

\subsection{Iterative Algorithm for Solving Power Allocation Subproblem}

In this subsection, we assume that $\alpha_{l, k}^{(i)}=1$, i.e., the $k$ th channel is allocated to the $l$ th link of the $i$ th SCR, and formulate the power allocation subproblem as follows.

$$
\begin{aligned}
& \max _{P_{l, k}^{(i)}} \eta_{l, k}^{(i)} \\
& \text { s.t. } \mathrm{C} 1: P_{l, k}^{(i)} \leq P_{l}^{(i, \max )}, \\
& \mathrm{C} 2: P_{l, k}^{(i)} \leq \frac{1}{g_{l, k}^{(i, \mathrm{p})}}\left(\frac{P_{k}^{(\mathrm{p})} h_{k}^{(\mathrm{p})}}{2^{\frac{R_{k}^{(\mathrm{p}, \mathrm{min})}}{W}}-\sigma^{2}}\right), \\
& \mathrm{C} 3: R_{l, k}^{(i)} \geq R^{(\mathrm{s}, \mathrm{min})} .
\end{aligned}
$$

The optimization problem formulated in (26) is a nonlinear fractional problem which can be transformed into a convex problem and solved using iterative algorithm. To solve the problem, we introduce variable $q$ and denote $q^{*}$ as the maximum energy efficiency of the $l$ th link of the $i$ th SCR when the $k$ th channel is allocated, i.e.,

$$
q^{*}=\frac{R_{l, k}^{(i)}}{P_{l, k}^{(i, *)}+P_{\text {cir }}}=\max _{P_{l, k}^{(i)}} \frac{R_{l, k}^{(i)}}{P_{l, k}^{(i)}+P_{\text {cir }}} .
$$

It can be proved that the maximum energy efficiency $q^{*}$ is achieved if and only if [11]

$$
R_{l, k}^{(i)}\left(P_{l, k}^{(i)}\right)-q\left(P_{l, k}^{(i)}+P_{\text {cir }}\right)=0
$$

Hence, solving the optimization formulated in (26) is equivalent to solving the following optimization problem:

$$
\begin{gathered}
\max _{q, P_{l, k}^{(i)}} R_{l, k}^{(i)}-q\left(P_{l, k}^{(i)}+P_{\text {cir }}\right) \\
\text { s.t. } \mathrm{C} 1 \sim \mathrm{C} 3 \mathrm{in}(26) .
\end{gathered}
$$

Applying iterative algorithm, the optimal energy efficiency $q^{*}$ and power allocation strategy $P_{l, k}^{(i, *)}$ can be obtained. The problem solving process can be summarized briefly: starting from an initial value of $q$, the locally optimal power allocation strategy can be obtained through applying traditional convex optimization tools, then the energy efficiency $q$ can be updated based on the obtained power solution; given the updated $q$, the power allocation process can be reconducted, the process continues until the algorithm converges and the optimal energy efficiency and the power allocation strategy can be obtained [11].

For a given $q$, the power allocation subproblem can be formulated as:

$$
\begin{aligned}
& \max _{P_{l, k}^{(i)}} R_{l, k}^{(i)}-q\left(P_{l, k}^{(i)}+P_{\text {cir }}\right) \\
& \text { s.t. C1 } \sim \text { C3 in }(26) .
\end{aligned}
$$

The optimization problem formulated in (30) is a constrained convex optimization problem which can be solved by applying Lagrangian method. The Lagrangian function 
can be formulated as:

$$
\begin{aligned}
& L\left(\lambda, \mu, \omega, P_{l, k}^{(i)}\right)= \\
& R_{l, k}^{(i)}-q\left(P_{l, k}^{(i)}+P_{\text {cir }}\right)-\lambda\left(P_{l, k}^{(i)}-P_{l}^{(i, \max )}\right) \\
& -\mu\left(P_{l, k}^{(i)}-Q_{l, k}^{(i)}\right)-\omega\left(R^{(\mathrm{s}, \min )}-R_{l, k}^{(i)}\right)
\end{aligned}
$$

where $\lambda, \mu, \omega$ are Lagrange multipliers, and

$$
Q_{l, k}^{(i)}=\frac{1}{g_{l, k}^{(i, \mathrm{p})}}\left(\frac{P_{k}^{(\mathrm{p})} h_{k}^{(\mathrm{p})}}{2^{\frac{R_{k}^{(\mathrm{p}, \mathrm{min})}}{W}}-1}-\sigma^{2}\right) .
$$

The optimization problem in (30) can be transformed into Lagrange dual problem:

$$
\begin{aligned}
& \min _{\lambda, \mu, \omega} \max _{P_{i, k}^{(i)}} L\left(\lambda, \mu, \omega, P_{i, k}^{(i)}\right) \\
& \text { s. t. } \lambda \geq 0, \mu \geq 0, \omega \geq 0 .
\end{aligned}
$$

The optimization problem formulated in (33) consists of two subproblems, i.e., internal maximum subproblem and external minimum subproblem, which can be solved iteratively. For a set of fixed Lagrange multipliers, the internal maximum subproblem can be solved to obtain the locally optimal power allocation strategy, which can then be applied to solve the external minimum subproblem to obtain the updated Lagrange multipliers.

The locally optimal power allocation strategy can be obtained by calculating the derivative of formulated Lagrange function with respect to $P_{i, k}^{(i)}$ and setting to zero, i.e.,

$$
\begin{aligned}
& \frac{\partial L\left(\lambda, \mu, \omega, P_{i, k}^{(i)}\right)}{\partial P_{l, k}^{(i)}}= \\
& W \frac{(1+\omega) h_{l, k}^{(i)}}{\ln 2\left(\sigma^{2}+P_{k}^{(\mathrm{p})} g_{l, k}^{(\mathrm{p}, i)}+P_{l, k}^{(i)} h_{l, k}^{(i)}\right)}-q-\lambda-\mu=0,
\end{aligned}
$$

we can obtain

$$
P_{l, k}^{(i, *)}=\left[\frac{(1+\omega) W}{(q+\lambda+\mu) \ln 2}-\frac{\sigma^{2}+P_{k}^{(p)} g_{l, k}^{(\mathrm{p}, i)}}{h_{l, k}^{(i)}}\right]^{+}
$$

where $[x]^{+}=\max \{x, 0\}$.

To solve the external minimum subproblem, we apply gradient descent algorithm to calculate the Lagrange multiplier [11]:

$$
\begin{gathered}
\lambda(t+1)=\left[\lambda(t)-\varepsilon_{1}\left(P_{l}^{(i, \max )}-P_{l, k}^{(i)}\right)\right]^{+}, \\
\omega(t+1)=\left[\omega(t)-\varepsilon_{2}\left(R_{l, k}^{(i)}-R^{(s, \min )}\right)\right]^{+}, \\
\mu(t+1)=\left[\mu(t)-\varepsilon_{3}\left(Q_{l, k}^{(i)}-P_{l, k}^{(i)}\right)\right]^{+}
\end{gathered}
$$

where $t$ denotes the iteration factor, $\varepsilon_{k}>0, k=1,2,3$, are defined as the iteration stepsize of $\lambda, \omega$ and $\mu$, respectively. The iteration process over Lagrange multipliers repeats until it achieves convergence.

\subsection{Channel Allocation Subproblem}

After solving the power allocation subproblem for the $l$ th hop of the $i$ th SCR on the $k$ th channel, we can obtain the local optimal power allocation strategy, i.e., $1 \leq l \leq L_{\min }$, $1 \leq k \leq K$, the optimal channel allocation subproblem can be formulated as follows.

$$
\begin{aligned}
& \max _{\substack{\alpha_{l, k}^{(i)} \\
\text { s. t. }}} \eta^{(i)}\left(P_{l, k}^{(i, *)} \sim \mathrm{C} 5 \text { in }(24) .\right.
\end{aligned}
$$

The optimal channel allocation strategy for the $l$ th hop of the $i$ th SCR can be obtained by comparing the energy efficiency on various channels and selecting the one offering the maximum energy efficiency, i.e.,

$$
\alpha_{l, k}^{(i, *)}=\arg \max \eta_{l, k}^{(i)}\left(P_{l, k}^{(i, *)}\right) .
$$

\section{SIMULATION RESULTS}

In this section, the performance of the proposed joint resource allocation and route selection algorithm is evaluated via simulation. The simulation scenario is a square region of which the width is 500 meters. The number of PUs and the licensed channels are both set to be 4 . The PUs, a pair of source and destination SUs, and multiple relay SUs are uniformly distributed in the simulation region and the number of relay SUs varies from 8 to 16 . In the simulation, the channel model between a transmitter and the corresponding receiver is assumed as $h=C d^{-2}$, where $C$ denotes the channel gain factor and $d$ denotes the distance between the transmitter and the receiver. Other system parameters chosen in the simulation are summarized in Table I.

Fig. 2 shows the energy efficiency versus the number of iterations for different numbers of the relay SUs. The maximum transmit power $P_{l}^{(i, \max )}$ is chosen as 0.2 in plotting the figure, the number of relays is denoted as $N_{\text {rs }}$. It can be observed that the energy efficiency converges within a small number of iterations.

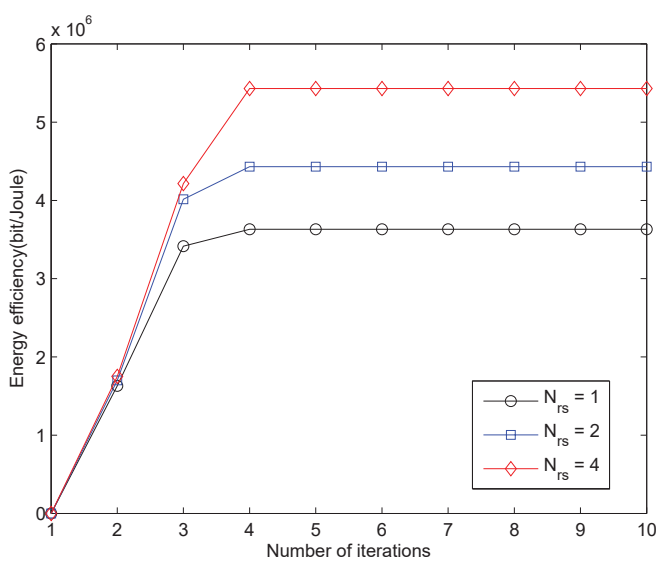

Figure 2: Energy efficiency versus the number of iterations 
Table 1: Simulation Parameter

\begin{tabular}{|l|l|}
\hline Parameter Name & Value \\
\hline PU Pairs & 4 \\
\hline Channel bandwidth W(HZ) & $10^{6}$ \\
\hline Maximum iteration number of $q$ & 10 \\
\hline Maximum Iteration number $T_{\max }$ & 5 \\
\hline Circuit Power & 0.2 \\
\hline Minimal data rate requirement (bit/s) & $10^{6}$ \\
\hline
\end{tabular}

Fig. 3 shows the energy efficiency versus the maximum transmit power for different numbers of the relays. To illustrate the performance of the proposed algorithm, we also plot the performance of a baseline algorithm. For baseline algorithm, we first apply the CSPF algorithm to find the shortest routes, then choose the optimal route which maximizes the transmission rate of the SU pair, instead of the energy efficiency. It can be seen from the figure that for small $P_{\max }$, the energy efficiency increases with the increase of $P_{\max }$, indicating a larger power threshold is desired for achieving the maximum energy efficiency. However, as $P_{\max }$ reaches to a certain value, the energy efficiency obtained from our proposed algorithm becomes a fixed value for the transmit power being less than $P_{\max }$ has resulted in the optimal energy efficiency, which will no longer vary with $P_{\max }$, however, the energy efficiency obtained form the baseline algorithm becomes decrease after reaching the maximum value. This is because the baseline algorithm aims at maximizing the data rate, hence may require high transmit power, resulting in low energy efficiency. Comparing the results obtained from the two algorithms, we can see that the proposed algorithm outperforms the baseline algorithm.

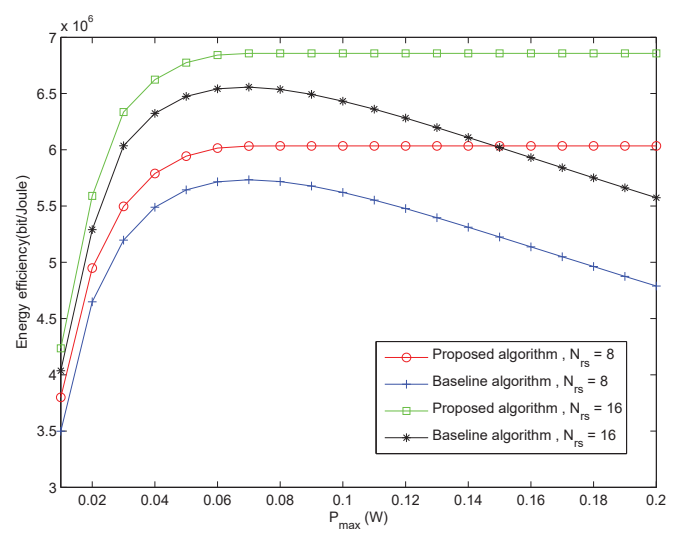

Figure 3: Energy efficiency versus $P_{\max }$

The energy efficiency versus the maximum transmit power for different circuit power consumption is shown in Fig. 4. For comparison, we plot the energy efficiency obtained from our proposed algorithm and the baseline algorithm. It can be seen from the figure that the energy efficiency obtained from both algorithms decreases with the increase of the circuit power consumption. Comparing the results obtained from the two algorithms, we can see that the proposed algorith$m$ offers higher energy efficiency than that of the baseline algorithm.

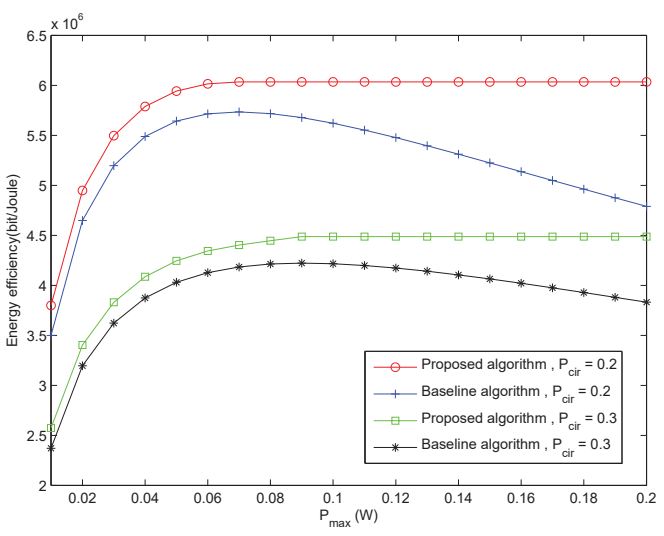

Figure 4: Number of hops versus $P_{\max }$

\section{CONCLUSION}

In this paper, to achieve the performance enhancement of multi-hop transmission in CRNs, an energy efficient CSPFbased joint resource allocation and route selection algorithm is proposed, which consists of two sub-algorithms, i.e., CSPFbased route selection sub-algorithm and energy efficiencybased resource allocation sub-algorithm. Specifically, we first apply CSPF-based route selection to obtain the SCRs, then, for each SCR, we formulate the optimal resource allocation problem as energy efficiency maximization problem and solve the problem by applying iterative algorithm and Lagrange dual method. Finally, the energy efficiency of the SCR is examined and the globally optimal route selection and resource allocation strategy is obtained which offers the maximal energy efficiency of the transmission route. Simulation results demonstrate the effectiveness of the proposed algorithm.

\section{ACKNOWLEDGEMENT}

This work is supported by the National Science and Technology Specific Project of China (2016ZX03001010-004), the special fund of Chongqing key laboratory (CSTC), and the project of Chongqing Municipal Education Commission (Kjz h11206).

\section{REFERENCES}

[1] J. Mitola and J. Maguire, G.Q., "Cognitive radio: making software radios more personal," IEEE Personal Communications, vol. 6, pp. 13-18, Aug 1999.

[2] A. Ewaisha and C. Tepedelenlioglu, "Delay optimal joint scheduling-and-power-control for cognitive radio uplinks," in 2016 IEEE Global Communications Conference (GLOBECOM), pp. 1-5, IEEE, Deceber 2016. 
[3] M. Zeng, G. I. Tsiropoulos, O. A. Dobre, and M. H. Ahmed, "Power allocation for cognitive radio networks employing nonorthogonal multiple access," in 2016 IEEE Global Communications Conference (GLOBECOM), pp. 1-5, IEEE, Deceber 2016.

[4] Y. Han, E. Ekici, H. Kremo, and O. Altintas, "Throughputefficient channel allocation algorithms in multi-channel cognitive vehicular networks," IEEE Transactions on Wireless Communications, vol. 16, pp. 757-770, February 2017.

[5] H. K. Boddapati, M. R. Bhatnagar, and S. Prakriya, "Ad-hoc relay selection protocols for multi-hop underlay cognitive radio networks," in 2016 IEEE Globecom Workshops (GC Wkshps), pp. 1-6, IEEE, Deceber 2016

[6] O. Georgiou, M. Z. Bocus, and S. Wang, "Distributed power allocation and channel access probability assignment for cognitive radio," in 2015 IEEE Global Communications Conference (GLOBECOM), pp. 1-6, IEEE, Deceber 2015

[7] R. Combes and A. Proutiere, "Dynamic rate and channel selection in cognitive radio systems," IEEE Journal on Selected Areas in Communications, vol. 33, pp. 910-921, May 2015.

[8] W. Yang and X. Zhao, "Robust relay selection and power allocation for ofdm-based cooperative cognitive radio networks," in 2016 IEEE Global Communications Conference (GLOBECOM), pp. 1-7, IEEE, Deceber 2016.

[9] R. M. Amini and Z. Dziong, "An economic framework for routing and channel allocation in cognitive wireless mesh networks," IEEE Transactions on Network and Service Management, vol. 11, pp. 188-203, December 2014

[10] A. A. El-Sherif and A. Mohamed, "Joint routing and resource allocation for delay minimization in cognitive radio based mesh networks," IEEE Transactions on Wireless Communications, vol. 13, pp. 186-197, December 2014.

[11] D. W. K. Ng, E. S. Lo, and R. Schober, "Energy-efficient resource allocation in SDMA systems with large numbers of base station antennas," in 2012 IEEE International Conference on Communications (ICC), pp. 4027-4032, IEEE, June 2012 